OPEN ACCESS

Edited by:

Arumugam R. Jayakumar, Miami VA Healthcare System,

United States

Reviewed by:

Faiçal Isbaine,

Emory University Hospital,

United States

Heena Kausar Shaik,

University at Buffalo, United States

*Correspondence:

Bonghee Lee

bhlee@gachon.ac.kr

${ }^{\dagger}$ These authors have contributed equally to this work

Specialty section: This article was submitted to

Neurodegeneration,

a section of the journal

Frontiers in Neuroscience

Received: 15 June 2021 Accepted: 01 September 2021 Published: 06 October 2021

Citation:

Kim HJ, Bayarsaikhan D, Lee J, Bayarsaikhan $G$ and Lee B (2021) Brain-Derived Neurotrophic Factor Secreting Human Mesenchymal Stem

Cells Improve Outcomes in Rett

Syndrome Mouse Models.

Front. Neurosci. 15:725398

doi: 10.3389/fnins.2021.725398

\section{Brain-Derived Neurotrophic Factor Secreting Human Mesenchymal Stem Cells Improve Outcomes in Rett Syndrome Mouse Models}

\author{
Hyo Jeong Kim ${ }^{1,2+}$, Delger Bayarsaikhan ${ }^{3 \dagger}$, Jaesuk Lee ${ }^{3,4}$, Govigerel Bayarsaikhan ${ }^{3}$ and \\ Bonghee Lee ${ }^{1,3 *}$ \\ ${ }^{1}$ Department of Anatomy \& Cell Biology, Gachon University College of Medicine, Incheon, South Korea, ${ }^{2}$ Department \\ of Pediatrics, Gil Medical Center, Gachon University College of Medicine, Incheon, South Korea, ${ }^{3}$ Center for Genomics \\ and Proteomics and Stem Cell Core Facility, Lee Gil Ya Cancer and Diabetes Institute, Gachon University, Incheon, \\ South Korea, ${ }^{4}$ Department of Chemistry, College of Natural Science, Seoul National University, Seoul, South Korea
}

Rett syndrome (RTT) is a severe X-linked dominant neurodevelopmental disorder caused by mutations in the methyl-CpG-binding protein 2 (MECP2) gene; MeCP2 regulates the expression of brain-derived neurotrophic factor (BDNF) and increasing BDNF levels ameliorates RTT symptoms. However, the clinical application of BDNF is limited, because of its short half-life and low penetrance across the blood-brain barrier. In this study, we generated BDNF-secreting mesenchymal stem cells (MSCs) from the human umbilical cord cells, using CRISPR-Cas9. We studied the effects of BDNF-MSCs in MECP2 knockout and MECP2-deficient mice. BDNF-MSCs upregulated the expression of BDNF, pAKT, and $\mathrm{pERK} 1 / 2$ and downregulated that of $\mathrm{pp} 38$, both in vitro and in vivo. In our in vivo experiments, BDNF-MSCs increased the body and brain weights in mice. BDNF-MSCs increased the neuronal cell numbers in the hippocampus, cortex, and striatum; in addition, they increased the number of synapses. BDNF-MSCs upregulated BDNF and the activity of BDNF downstream effectors, such as PAKT and pERK 1/2; this upregulation was persistent. In conclusion, BDNF-MSCs generated using CRISPR-Cas9 could be a therapeutic strategy for treating $\mathrm{RTT}$.

Keywords: Rett syndrome, MECP2, brain-derived neurotrophic factor, CRISPR-Cas system, transplantation, mesenchymal stem cell

\section{INTRODUCTION}

Rett syndrome (RTT) is a severe X-linked dominant neurodevelopmental disorder that affects approximately 1 in 10,000 female infants. It is categorized into classical and atypical RTT. Majority (95\%) of the cases of classical type RTT are caused by de novo mutations in the MECP2 gene located on the X-chromosome (Pejhan and Rastegar, 2021). Female RTT patients develop normally during the first 6 months of life, but thereafter show regression in motor skills, language, and cognition. Characteristics of RTT include slower growth in head circumference, stereotypic hand movements, and loss of purposeful hand skills. Seizures, ataxia, and breathing disturbances are also common (Percy et al., 2010). MECP2 gene mutations affect male more severely than female, leading to early fetal death or severe neonatal encephalopathy (Villard, 2007). MECP2 plays a crucial role in both activation and inhibition/silencing of genes. Brain-derived neurotrophic factor $(B D N F)$ is an established target gene of $M E C P 2$, and the correlation between these two genes in RTT is widely studied. Deficiency of $M E C P 2$, through phosphorylation of the $M E C P 2$ gene or epigenetic modifications, 
leads to a reduction in the expression, translation, and stability of in vivo BDNF (Damen and Heumann, 2013). Animal models of RTT show that BDNF is downregulated in the brain, serum, and cerebrospinal fluid; BDNF deficiency leads to symptoms and outcomes similar to that of de novo mutations in the MECP2 gene. Overexpression of BDNF against MECP2 deficiency in RTT model mice suggests that BDNF could be an alternative therapeutic for RTT (Vanhala et al., 1998; Riikonen, 2003; Abuhatzira et al., 2007).

There is no cure for RTT; however, results from preclinical studies suggest a wide range of therapeutic strategies for RTT, including direct administration of supplements such as MECP2 and BDNF to relieve symptoms (Luoni et al., 2020; Collins et al., 2021). Drugs that increase the levels of BDNF such as glatiramer acetate, known as a multiple sclerosis immune modulator, could be used to recover neuronal function by stimulating the serotonin receptor 7 in conjunction with inhibiting PTP1B, which blocks the TRKB receptor of BDNF (Krishnan et al., 2015; Napoletani et al., 2021; Vigli et al., 2021). However, evaluating their safety and efficacy are critical, because of dosage-sensitive secondary responses, poor stability, and in vivo penetration abilities (Tsai, 2012; Lombardi et al., 2017). Advances in gene engineering technologies, such as TALEN and CRISPR, enable successful and continuous introduction of therapeutic molecules with a short half-life, for alleviating the pathogenesis of genetic disorders (Bayarsaikhan et al., 2020; Lee et al., 2020).

We hypothesized that a combination of therapeutic strategies, such as targeted PTP1B injection followed by continuous overexpression of BDNF via local injection of CRISPR/Cas9 - edited MSCs could be a potential strategy for managing RTT. We believe that this would significantly eliminate the limitations in the clinical translation of previously developed methods. To overcome the limitations of BDNF administration, we generated BDNF-secreting mesenchymal stem cells (MSCs) from the human umbilical cord (UC) cells using the CRISPR/Cas9 system. A continuous supply of BDNF via the secretion from MSCs could ameliorate the symptoms of RTT by restoring neuronal size, morphology, and synaptic function. This study investigated whether BDNFsecreting MSCs generated by CRISPR/Cas9 could reverse the neuronal pathology in MECP2-deficient RTT mouse models and in neurons that underwent MECP2 knockdown. We also aimed to identify the downstream mechanisms of MeCP2 and BDNF.

\section{MATERIALS AND METHODS}

\section{Cell Culture}

Umbilical cord blood-driven MSCs (UCB-MSCs) (Medipost) were grown in $\alpha$-MEM medium (DMEM, Gibco) with $10 \%$ fetal bovine serum (FBS, Gibco) and 1\% penicillin streptomycin (Sigma-Aldrich). The cells were kept in an incubator under humidified conditions at $37^{\circ} \mathrm{C}$ and $5 \% \mathrm{CO}_{2}$. The culture medium was changed three times per week, and cells were sub-cultured at $80 \%$ confluence.

\section{Generation of Brain-Derived Neurotrophic Factor-Secreting Umbilical Cord Blood-Driven-Mesenchymal Stem Cells}

To generate BDNF-secreting UCB-MSCs, transfection was performed using the CRISPR/Cas9 ribonucleoprotein system (ToolGen, Inc.), which targets a safe harbor site known as adeno-associated virus site 1 (AAVS1). The donor vector was purchased from Sigma Aldrich (pZDonor-AAVS1 Puromycin vector, PZD0020), and the FLAG-tagged human BDNF encoding sequence was cloned into the multiple cloning site. Nucleofection was performed using a $4 \mathrm{D}$ nucleofector (Lonza) by transfecting $15 \mu \mathrm{g}$ of Cas 9 protein, $20 \mu \mathrm{g}$ of gRNA (GGGGCCACTAGGGACAGGAT), and $1 \mu \mathrm{g}$ of donor DNA into $8 \times 10^{5}$ UCB-MSCs. Transfected cells were incubated at $37^{\circ} \mathrm{C}$ for 3 days to stabilize cell conditions. On day 4 , the cells were treated with $1 \mu \mathrm{g} / \mathrm{ml}$ puromycin for 5 days to select the positive cells (with successful genome integration of the target sequence). Media was changed once in every 2 days and the culture was maintained until cell confluence was reached and it was ready for injection.

\section{MeCP2 Gene Silencing by siRNA}

SHSY-5Y neuroblastoma cells were seeded at a density of $5 \times 10^{5}$ cells per well of a 6-well culture dish. After $24 \mathrm{~h}$, cells were transfected with MeCP2 siRNA (QIAGEN, HS_MeCP2_7, SI02664893, target sequence ACGGAGCGGATTGCAAAGCAA) at final concentrations of 5 and $200 \mathrm{nM}$ using the FuGene- 6 transfection reagent (Roche), according to the manufacturer's instructions. Fortyeight hours after transfection, cells were harvested, and RNA was isolated using the RNAesy mini kit (QIAGEN; 74104). MeCP2 expression was quantified using RT-PCR.

\section{Rett Syndrome Mouse Model}

The animal experiments were performed at the Center of Animal Care and Use (CACU), Lee Gil Ya Cancer and Diabetes Institute of GACHON University. Five-week-old MECP2-deficient male mice $\left(\right.$ Mecp2 ${ }^{-/ y}$ ) (The Jackson Laboratory, stock no: 003890, $\operatorname{Mecp} 2^{\mathrm{TM} 1}{ }^{1 \text { Bird }}$ ) and 5-week-old male C57BL/6N mice were randomly divided and housed with five mice per cage in a temperature-controlled environment with a $12 \mathrm{~h}$ light/dark cycle. The mice had free access to food and water. All animal protocols described in this study were approved by the CACU Animal Center Ethics Board (LCDI-2018-008).

\section{Stereotaxic Surgery and Tissue Preparation}

When MECP2-deficient mice were 6 weeks old, the animals were divided into three groups: RTT, BDNF-protein-injected, and BDNF-secreting UCB-MSC-injected groups. In addition, the PTP1b inhibitor CPT157633 (Sigma-Aldrich, 539741) was intraperitoneally injected into the rhBDNF/BDNF-MSC injected mice every other day at a dose of $5 \mathrm{mg} / \mathrm{kg}$ to activate the BDNF receptor (TRKB) prior to the 2-week period of cell transplantation experiments. At 8 weeks of age, the RTT model 
animals were sacrificed (Krishnan et al., 2015). Animals were anesthetized using isoflurane prior to the surgical procedures. A lateral injection was performed into the left ventricle of the brain (anterior: $0.3 \mathrm{~mm}$, lateral: $1.0 \mathrm{~mm}$, dorsal: $3.0 \mathrm{~mm}$ from Bregma). For protein injection, $500 \mathrm{ng}$ of BDNF protein (Abcam, ab9794) was prepared in $10 \mu \mathrm{L}$ of saline. For the UCB-MSC injection, $5 \times 10^{5}$ cells were prepared in $10 \mu \mathrm{l}$ of $\alpha$-MEM medium without FBS and antibiotics. Perfusion was performed under anesthesia by injecting the heart with $50 \mathrm{~mL}$ of $1 \times$ PBS followed by $50 \mathrm{~mL}$ of cold fixative consisting of $4 \%$ paraformaldehyde (PFA).

\section{Enzyme-Linked Immunosorbent Assay}

The total BDNF secreted in the cell culture medium was quantified using the Human BDNF Simple Step ELISA kit (Abcam; ab212166), according to the manufacturer's instructions.

\section{Western Blot Analysis}

Brain tissue and total cell lysates were prepared for western blotting by adding the EzRIPA lysis buffer (ATTO; WSE-7420) and $1 \times$ protease/phosphatase inhibitor (ATTO; WSE-7420), followed by sonication. The tissue lysate was centrifuged at $14,000 \times g$ for $20 \mathrm{~min}$ at $4^{\circ} \mathrm{C}$, and the supernatant was collected. Total protein concentration was measured using the BCA protein assay (Life Technologies; 23227), according to the manufacturer's instructions. Equal amounts $(20 \mu \mathrm{g})$ of protein were electrophoresed on $10 \%$ polyacrylamide gels (Life Technologies; WG1203BOX) and transferred to a PVDF membrane (Millipore; GSWP01300). Proteins were detected using protein-specific antibodies (Table 1); ECL detection reagent (Life Technologies; 32109) was used to visualize the immunoreactive proteins on the membrane.

\section{Polymerase Chain Reaction}

RT-PCR was performed to determine the expression levels of target genes. Total RNA was isolated using RNAesy mini kit (QIAGEN; 74104) from the total cell lysate, according to the manufacturer's instructions. RNA was converted to cDNA using a cDNA synthesis kit (Takara, 6110A) and used for RT-PCR experiments with target-specific primers (Table 1).

\section{Immunostaining}

Mouse brain tissues were deparaffinized in xylene for $10 \mathrm{~min}$ at room temperature and hydrated using a graded ethanol series (100\% ethanol for $5 \mathrm{~min}$, 90\% ethanol for $5 \mathrm{~min}, 80 \%$ ethanol for $5 \mathrm{~min}$, and $70 \%$ ethanol for $5 \mathrm{~min}$ ). Briefly, the remaining ethanol was washed with distilled water. Before the incubation

TABLE 1 | List of primer used in this study.

\begin{tabular}{lcccc}
\hline Gene & Direction & Sequence & Tm & Size \\
\hline GAPDH & Forward & ACCCAGAAGACTGTGGATGG & $59^{\circ} \mathrm{C}$ & $415 \mathrm{bp}$ \\
GAPDH & Reverse & TGCTGTAGCCAAATCGTTG & $55^{\circ} \mathrm{C}$ & $415 \mathrm{bp}$ \\
MeCP2 & Forward & CAGGTCATGGTGATCAAACG & $56^{\circ} \mathrm{C}$ & $278 \mathrm{bp}$ \\
MeCP2 & Reverse & AGTCCTTCCCGCTCTTCTC & $59^{\circ} \mathrm{C}$ & $278 \mathrm{bp}$
\end{tabular}

with antibody, tissues were heated in antigen retrieval buffer and washed in $1 \times$ PBS for five times.

The tissue samples were incubated with primary antibodies (Table 2) overnight at $4^{\circ} \mathrm{C}$. The excess antibodies were removed by washing with $1 \times$ PBS thrice, followed by incubation with a secondary fluorescent antibody (Table 1) at room temperature for $1 \mathrm{~h}$. Nuclei were counterstained with DAPI (4'6-diamino2-phenylindole; $1 \mu \mathrm{g} / \mathrm{ml}$, Invitrogen, D1306) for $20 \mathrm{~s}$ at room temperature. After washing with PBS, cover slides were mounted using Vectashield mounting media (Vector Laboratories, H1000) and images were analyzed using an LSM 710 confocal microscope (Carl Zeiss).

\section{Cresyl Violet Staining}

Paraffin-embedded mouse brain tissues were deparaffinized in xylene for $10 \mathrm{~min}$ at room temperature and hydrated using a graded ethanol series (100\% ethanol for $5 \mathrm{~min}$, 95\% ethanol for $5 \mathrm{~min}$, and $70 \%$ ethanol for $5 \mathrm{~min}$ ). The brain tissue was washed with distilled water and was stained with $0.5 \%$ cresyl violet acetate (Sigma-Aldrich; 10510-54-0) solution for $8 \mathrm{~min}$ and washed using a graded ethanol series $(70 \%, 30 \mathrm{~s} ; 80 \%, 30 \mathrm{~s}$; 95\%, $30 \mathrm{~s} ; 100 \%, 30 \mathrm{~s}$; and 100\%, $5 \mathrm{~min}$ ). The stained slides were mounted with DPX mounting medium (Sigma-Aldrich; 06522) for microscopic image analysis.

\section{Densitometry}

The intensity of each immunoreactivity band was determined using Image J gel digitizing software.

\section{Statistical Analysis}

All data represent results from at least three independent experiments. All experimental data are presented as mean \pm standard deviation (SD). Statistical significance was determined using Student's $t$-test, and a threshold of $P \leq 0.05$ was considered significant $\left(^{*}: p<0.05\right.$, ${ }^{* *}: p<0.01$, $* * *: p<0.001)$.

\section{RESULTS}

\section{Overexpression of Brain-Derived Neurotrophic Factor in CRISPR/Cas9 Mediated Mesenchymal Stem Cells}

Immunocytochemistry analysis was performed to validate and distinguish the expression of gene engineered BDNF from that of the naturally occurring proteins using FLAG antibodies (Figures 1A,B). The positive expression of FLAG was observed in both the conditioned medium and the cell lysate of MSCs transfected with pzDonor, but there was no FLAG expression in mock transfected MSCs (Figures 1C,D).

After validating the overexpression of BDNF in the genetically modified MSCs, we performed ELISA to measure the amount of secreted BDNF. We sampled the conditioned medium of BDNFtransfected MSCs and wild-type MSCs, which were maintained at comparable confluency and culture duration. The BDNF secretion level was 5.85 times higher in the medium with the cells 
TABLE 2 | List of antibodies used in this study.

\begin{tabular}{|c|c|c|c|c|}
\hline Antigen & Host & Company (Cat. No) & Application IHC WB & \\
\hline MeCP2 & Rabbit & Cell signaling (3456S) & & $1: 1000$ \\
\hline Map2 & Chicken & Abcam (ab5392) & $1: 200$ & \\
\hline Synapsin & Rabbit & Abcam (ab274430) & $1: 200$ & \\
\hline Akt & Rabbit & Cell signaling (9272S) & & \\
\hline pAkt & Rabbit & Cell signaling (9271S) & & \\
\hline FLAG & Rabbit & Sigma-aldrich (F7425) & & $1: 1000$ \\
\hline p38 & Rabbit & Cell signaling (9212L) & $1: 100$ & $1: 1000$ \\
\hline pp38 & Rabbit & Cell signaling (9211S) & $1: 100$ & $1: 1000$ \\
\hline $\mathrm{ERK} 1 / 2$ & Rabbit & Cell signaling (9102S) & $1: 100$ & $1: 1000$ \\
\hline $\mathrm{pERK} 1 / 2$ & Rabbit & Cell signaling (4377S) & $1: 100$ & $1: 1000$ \\
\hline B-Actin & Rabbit & Abcam (ab8227) & $1: 100$ & $1: 1000$ \\
\hline Peroxidase labeled anti-rabbit lgG & Rabbit & Vector (PI 1000) & & $1: 5000$ \\
\hline Alexa Fluor 555 donkey anti-rabbit lgG & Rabbit & Invitrogen (A31572) & $1: 500$ & \\
\hline Alexa Fluor 488 donkey anti-chicken lgG & Donkey & Invitrogen (A11001) & $1: 500$ & \\
\hline
\end{tabular}

A

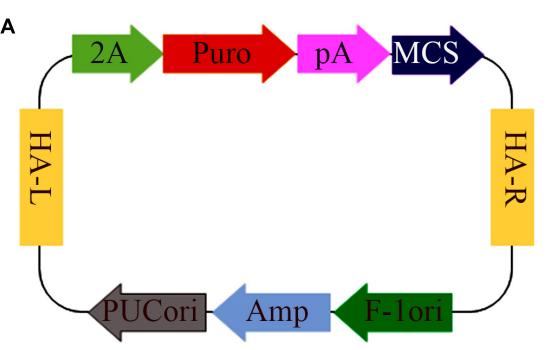

D

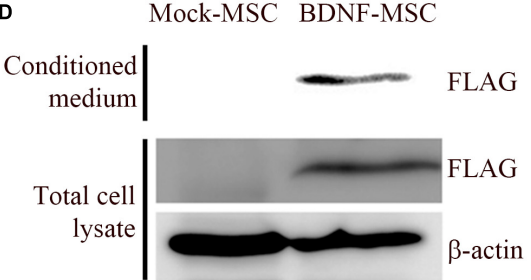

B -HA-L-Efl-a human BDNF -Poly A -HA-R -

c Marker Mock-MSC BDNF-MSC

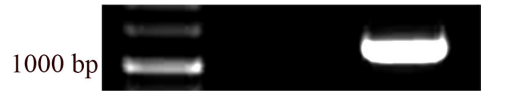

E

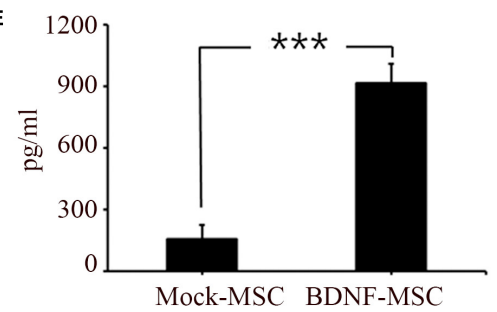

FIGURE 1 | Generation and characterization of BDNF secreting UCB-MSC. (A) pZDonor AAVS1 Puromycin vector. (B) Cloning of BDNF coding sequences, EF1 $\alpha$ promotor, and poly A tail. (C) Genome integration confirmed using Junction PCR with genomic DNA of UCB-MSCs, which were transfected with mock and BDNF containing pZDonor-AAVS1 vector. (D) Conditioned media and cell lysates from BDNF-secreting UC-MSCs tested using western blotting with FLAG antibodies. Only the transfection-derived BDNF protein can be detected using this method. (E) BDNF levels quantified using ELISA with BDNF antibodies. ${ }^{\star \star *} p<0.001$.

harboring gene edited MSCs, compared to that in the medium with wild-type MSCs (Figure 1E). We detected $914 \mathrm{pg} / \mathrm{ml}$ of BDNF in the conditioned medium from BDNF-MSCs and $156 \mathrm{pg} / \mathrm{mL}(p=0.001$; Figure 2B), from that of mock MSCs.

\section{In vitro Assay for Therapeutic Effects of Brain-Derived Neurotrophic Factor-Mesenchymal Stem Cells on Rett Syndrome-Modeled Cell Line} Cellular Model for Rett Syndrome

To evaluate the therapeutic effect of BDNF overexpressing MSCs, we generated a cellular model of RTT through siRNAmediated silencing of the MECP2 in the SHSY-5Y cell line. For silencing the MECP2 in the neuroblastoma cells, the efficacy of the siRNA at two different concentrations, 5 and $200 \mathrm{nM}$, were assessed using PCR analysis and immunohistochemical staining (Figures 3A,B). In PCR analysis, the GAPDH gene was used as the housekeeping gene. Quantitative analysis of MECP2 protein expression in the immunohistochemical staining was performed using ImageJ software. The expression of MECP2 in the neuroblastoma cells decreased by 6 - and 4.5 -fold following transfection with 5 and $200 \mathrm{nM}$ of siMeCP2, respectively. The lower dose was more efficient than the high dose of siRNA, without severely harming the cells; therefore, for further experiments, we used $5 \mathrm{nM}$ of siRNA. The co-localization of DAPI and MeCP2 was remarkably reduced in siMECP2 treated cells, compared to that in wild type cells, indicating the successful silencing of the MECP2 gene in siRNA-transfected cells (Figure 3C). 

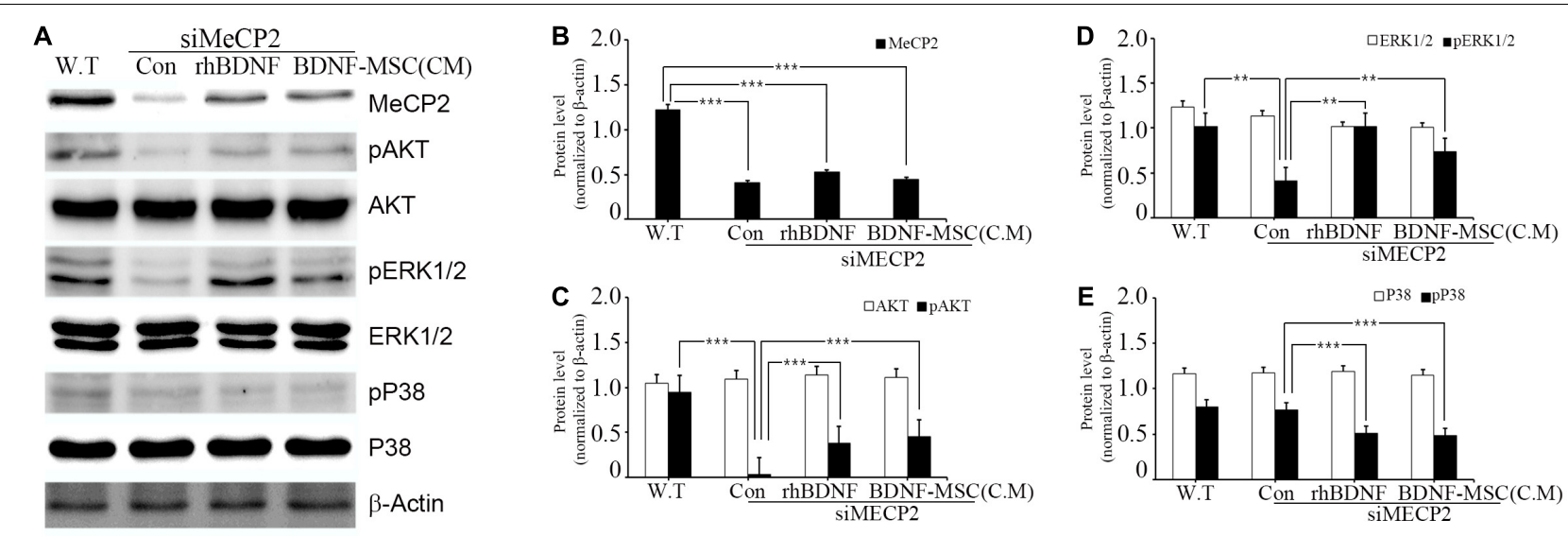

FIGURE 2 | Relative levels of MeCP2, AKT, and MAPK following rhBDNF or BDNF-MSC(CM) treatment in MeCP2 silenced cells. (A) Immunoblot analysis of the MeCP2 silenced cell lysates following rhBDNF or BDNF-MSC(CM) treatment. (B-E) Densitometry analyses of MAPK proteins evaluated using the Image-J software. Conditioned media from the control, siMeCP2, BDNF-treated, and BDNF-MSC-treated groups were tested. The phosphorylation of AKT and MAPK (ERK1/2 and p38) indicates changes in cell signaling. $\beta$-actin was used as the standard. ${ }^{* \star} p<0.01,{ }^{\star \star \star} p<0.001$.
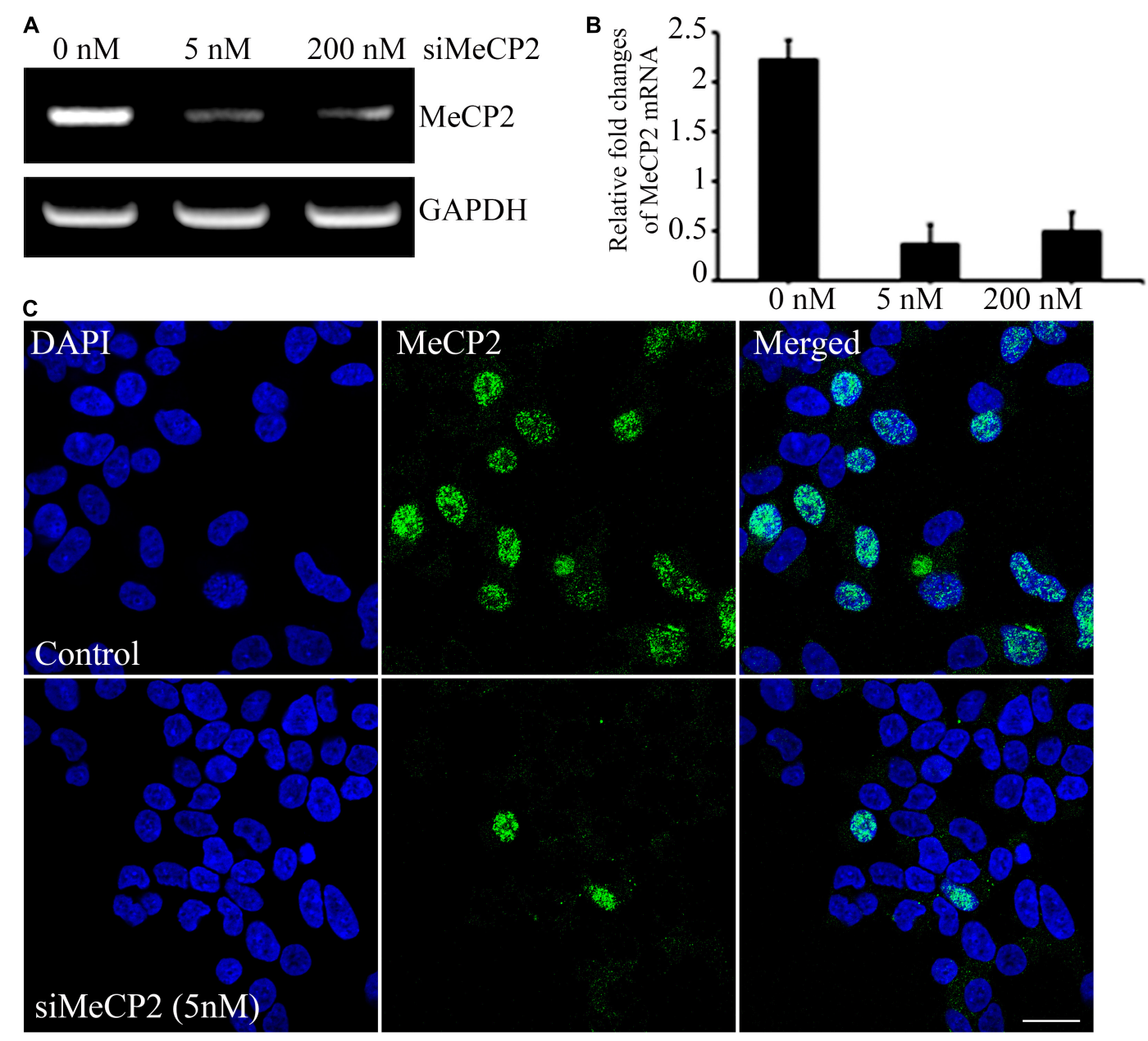

FIGURE 3 | Efficiency of MeCP2 silencing in SHSY-5Y neurons. (A) MeCP2 expression levels in knockdown cells, assessed using PCR, following treatment with different concentrations of MeCP2 siRNA. (B) Relative changes in expression are presented in a bar graph. (C) Immunohistochemistry for assessing the presence and localization of MeCP2. The cells were dual stained for the nucleus (blue) and MeCP2 (green) and observed using confocal microscopy. Scale bar $=50 \mu \mathrm{m}$. 


\section{A}

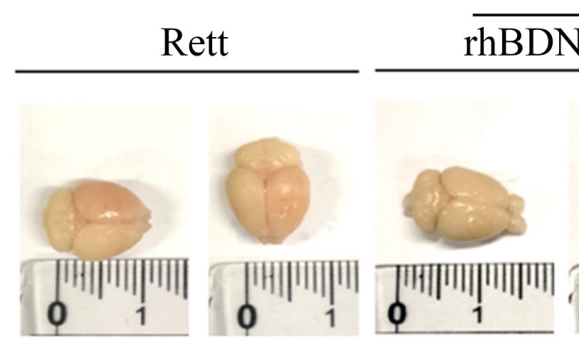

PTP1b inhibitor
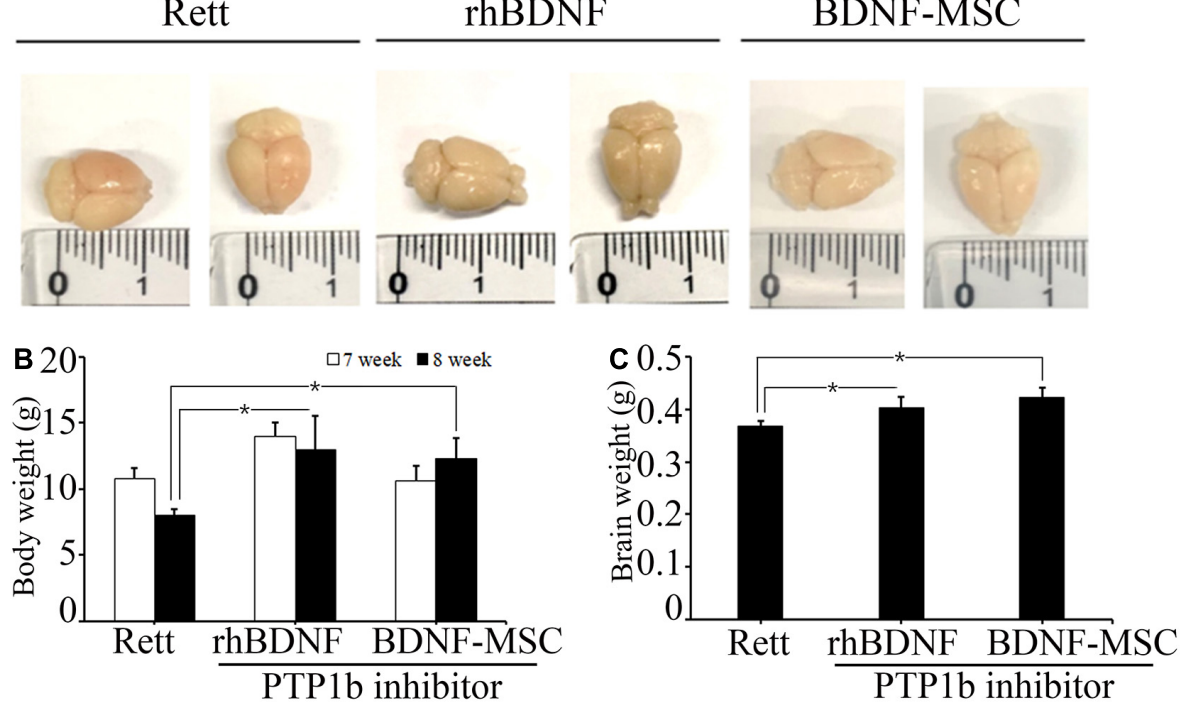

FIGURE 4 | Comparison of body and brain weights. (A) Stereotaxic surgery was performed at 7 weeks and brain samples were collected at 8 weeks of age. Brain size was measured after fixation with 4\% PFA. (B) The average body weights of the animals at 7 and 8 weeks of age. (C) Brain weight represented in a bar graph. ${ }^{*} p<0.05$.

\section{Effects of Brain-Derived Neurotrophic Factor Overexpression}

The specific affinity of BDNF toward tropomyosin-related kinase $\mathrm{B}$ (TRKB) leads to the downregulation of numerous pathways, such as mitogen-activated protein kinase (MAPK) and phosphatidyl-inositol 3-kinase (PI3K)/protein kinase B (AKT), which promote cell growth, differentiation, survival, and synaptic plasticity of the cell. Therefore, we aimed to investigate whether BDNF expression influenced the downregulation of these pathways. In addition, we examined the influence of BDNF expression on the survival of MECP2 silenced neuroblastoma cells. Wild type SHSY-5Y was used as the control for the RTT cellular model, in which the MECP2 gene was silenced. They were treated using either rhBDNF or the conditioned medium of BDNF overexpressing MSCs. The BDNF levels in the conditioned medium was determined using ELISA and, an equal amount of rhBDNF was applied. Western blot analysis demonstrated that the expression of $M e C P 2, p A K T, p E R K 1 / 2$, and $p p 38$ was downregulated in the MeCP2 knockdown cells, compared to that in the control cells (Figure 2A). The BDNF-treated and BDNF-MSCtreated groups were treated with a PTP1B (protein tyrosine phosphatase $1 \mathrm{~B}$ ) inhibitor. PTP1B targets the TRKB receptor and functions as a negative regulator of BDNF signaling. Therefore, we applied a PTP1B inhibitor to prevent the weakening of BDNF signaling. Both BDNF-treated and BDNFMSC-treated groups had increased levels of pAKT and pERK $1 / 2$ and decreased levels of pp38, compared to that in control (Figures 2B-E).

\section{In vivo Testing of Therapeutic Effects of Brain-Derived Neurotrophic Factor Overexpression via Mesenchymal Stem Cells in Rett Syndrome \\ Brain-Derived Neurotrophic Factor-Mesenchymal Stem Cells Prevent Loss of Body Weight and Brain Weight in MECP2 Knockout Mice}

We evaluated the therapeutic effects of BDNF secreting MSCs in RTT animal models at the tissue level. The MECP2 knockout mice models were transplanted with BDNF-overexpressing MSCs at an animal age of 6 weeks via surgical procedures and their body weight and brain weights were measured (Figure 4A). MECP2-deficient mice gradually lost weight and experienced frequent ataxia over time. There was no loss of body weight in the BDNF-MSC-treated groups, and there was statistically significant difference between the saline injected group and the BDNF-MSCtreated group after 2 -weeks of therapy $(p=0.048)$ (Figure $4 \mathbf{B})$. The mice were sacrificed 2 weeks after injecting either rhBDNF or BDNF overexpressing MSCs and their brain size and brain weight were measured. Among the three groups of RTT model mice, which were injected with saline solution, rhBDNF, or BDNF overexpressing MSCs, the control group showed the smallest brain size and brain weight, while the largest brain size and brain weight was found in the BDNF-MSC-treated groups (Figure 4C). Therefore, intraperitoneal application of BDNF promoted cell survival and enhanced the regeneration of brain tissue. The constitutive in vivo secretion in the brain ventricle prevented the loss of brain weight and size in MECP2 knockout mouse models 


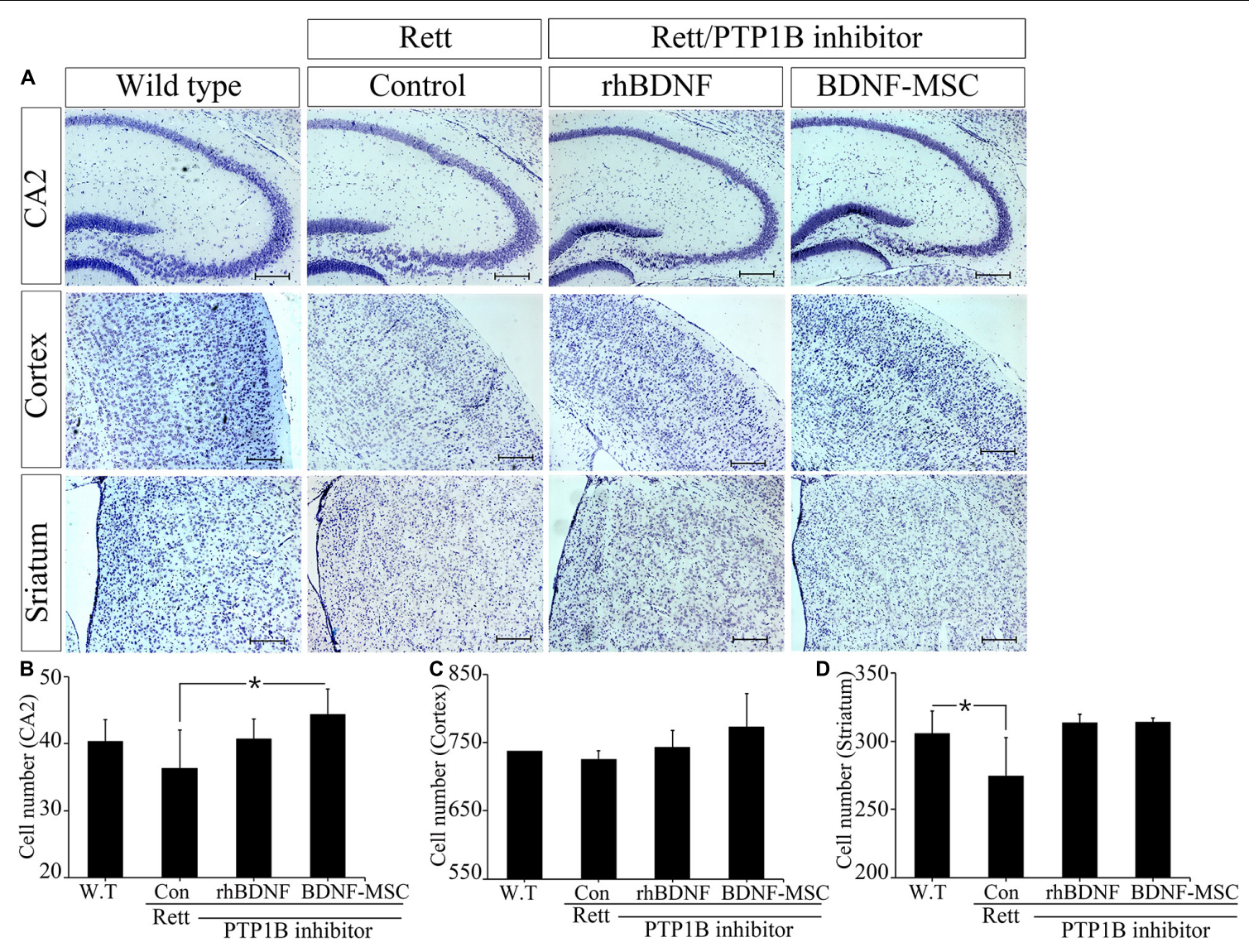

FIGURE 5 | Improved neurogenesis following BDNF-MSC treatment. (A) The samples from wild type, MECP2 knockout (Control-Rett), MECP2 knockout co-treated with BDNF/PTP1b inhibitor or BDNF-MSC/PTP1b groups were stained with cresyl violet. The cell numbers for each group were counted in three different areas of the brain: (B) CA2 of the hippocampus, (C) cortex, and (D) striatum. Cell numbers were counted using the ImageJ software and they are presented in the graphs. ${ }^{*} p<0.05$.

more efficiently, when compared to that using the synthetic form of the protein.

\section{Brain-Derived Neurotrophic Factor-Mesenchymal Stem Cells Promotes Growth of Neuronal Cells in the MECP2 Knockout Mice}

We narrowed the focus to the cellular level to understand how the brain weight and size were influenced by treatment with BDNF in the RTT model mice. Tissues sampled from the CA2 of the hippocampus, cortex, and striatum of the experimental animals from the four groups, including wild type mice (control) and MECP2 knockout mice treated with saline, rhBDNF, or BDNF-MSCs were stained with cresyl violet (Figure 5). Analysis using Image $\mathrm{J}$ software demonstrated that there was significant difference between the number of neurons in the CA2 of hippocampus of wild type and MECP2 deficient mice $(p=0.001)$. The number of neurons in the hippocampus of wild type mice and MECP2 knockout mice were $38.7 \pm 5.5$ and $36.3 \pm 5.7$, respectively, at 8 weeks of age. After transplantation of rhBDNFor BDNF-secreting MSCs into RTT model mice, the number of neurons increased to $40.7 \pm 3.1$ and $44.3 \pm 3.8$, respectively. These results suggest that RTT induced a reduction in the number of neurons and the neurogenesis in the hippocampus. However, these tend to improve in the presence of BDNF at the age between 6 and 8 weeks in MECP2 knockout mice. BDNFMSC treatment was more efficient than the injection of the recombinant form of the protein.

There was no difference in the number of neuronal cells in the cortex, between the control group and RTT group, which was $737.3 \pm 7.8$ and $725.7 \pm 12.2$, respectively. However, after injection of either rhBDNF or BDNF overexpressing MSCs, the number of cells gradually increased to $743.3 \pm 24.0$ and $773.0 \pm 49.0$, respectively. Therefore, BDNF increased neurogenesis in the cortex, and application of an in vivo expression technique for BDNF protein was more efficient in promoting neuronal cell growth in the cortex of $M E C P 2$ deficient mice.

We analyzed neurogenesis in the striatum of the brain samples obtained from experimental animals. The self-renewal capacity of the striatum neurons was relatively less in the MECP2 deficient mice, compared to that in the wild type mice. Two weeks after rhBDNF and BDNF-MSC injection, the number of neurons were 

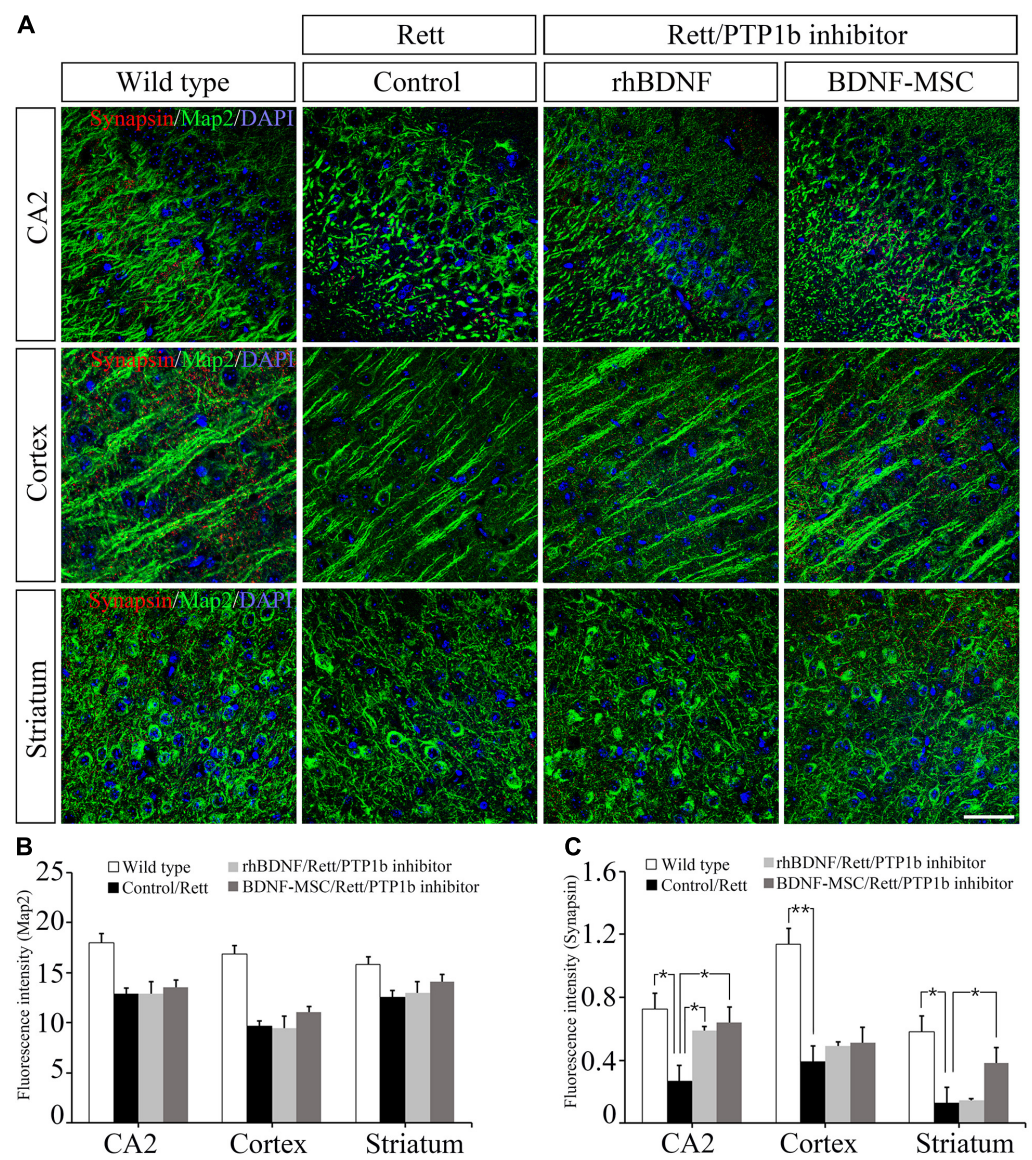

FIGURE 6 | Immunostaining analysis for detection of synapses. (A) Immunostaining confocal image showing expression of Map2 (green), synapses (red), and DAPI (blue) in mouse brain tissue. The expression intensity of (B) Map2 and $\mathbf{( C )}$ synapses were measured using Image $\mathrm{J}$ software. Scale bar $=50 \mu \mathrm{m}{ }^{*} p<0.05$, 
$313.3 \pm 6.7$ and $314.0 \pm 3.0$, respectively, and which were higher than that of saline injection group $(274.7 \pm 28.0)$. However, wild type mice had an average of $305.7 \pm 16.3$ striatal neurons. The neurons in the striatum were activated by rhBDNF and BDNFMSCs, similar to that in the hippocampal and cortical regions.

\section{Brain-Derived Neurotrophic Factor-Mesenchymal Stem Cells Treatment Increased Synapses in MECP2 Knockout Mice}

Two weeks post-injection of BDNF/BDNF-MSCs, immunostaining was performed on mouse brain tissues sampled from CA2 of the hippocampus, cortex, and striatum. Dysfunction of the X-linked MECP2 gene leads to diminished signaling in the junctions between the neurons located in the CA2 hippocampus, cortex, and striatum regions of the brain (Figure 6A). There were no considerable differences in the neurons responding to the MAP2 marker, among the MECP2 knockout mice injected with saline, rhBDNF, and BDNF-MSCs; however, they showed considerable reductions when compared to that in the wild type mice (Figure 6B).

We evaluated whether the presence of BDNF and deficiency of PTP1B affects the synaptic abilities of whole neurons located at different distances from the initial injection site in the brain. BDNF expressing MSCs highly altered the synaptic abilities of striatal neurons, and there were alterations in the CA2 hippocampal and cortical neurons, which were proportional to the distance from the injection site (Figure 6C).

\section{Brain-Derived Neurotrophic Factor-Mesenchymal Stem Cells Treatment Increased pAKT and pERK 1/2 and Decreased pp38 Expression in MECP2 Knockout Mice}

We investigated the relationship between the expression of $\mathrm{BDNF}$ and its downstream signaling pathways in MECP2 knockout mice using western blotting. There was no expression of MECP2 in the RTT model mice, while wild type mice had a high expression of MECP2 (Figure 7A). Analysis of the expression of total and phosphorylated forms of AKT, ERK1/2, and p38 indicated that phosphorylation of AKT and ERK1/2 increased and that of $\mathrm{p} 38$ decreased with the inhibition of PTP1B gene and the transplantation of BDNF protein in $M E C P 2$ knockout mice, compared to that in the wild type or MECP2 knockout mice injected with saline (Figures 7AE). The comparison between groups treated with rhBDNF and BDNF-MSCs showed that their phosphorylation effects were similar for pERK1/2 (Figure 7C). However, transplantation of BDNF-MSCs resulted in a higher phosphorylation of pAKT and a decreased expression of pP38 (Figures 7D,E). These results suggest that a continuous in vivo expression of BDNF could have better therapeutic effects, for improving the growth and functional activity of neurons during the pathogenesis of RTT.

\section{DISCUSSION}

We cloned a $2.1 \mathrm{~kb}$ insert containing the BDNF gene into the AAVS1 site of UC-MSCs, using the CRISPR/Cas9 system. Gene engineering could increase the secretion of the BDNF by at least 5.85 -folds, compared to that in wild type cells. BDNF augmentation could be performed using various carriers, including viruses and nanoparticles (Gao et al., 2016; Lopes et al., 2017). This study introduced a more efficient and safer carrier, the MSCs. Delivering BDNF via MSCs overcomes the limitations of poor diffusion into the bloodbrain barrier and short half-life, when compared to the use of recombinant BDNF (Khalin et al., 2016). Inactivation of the TRKB receptor results in a gradual decrease in the BDNF levels in the entire brain during the pathogenesis of RTT. In mammals, BDNF plays a crucial role in the survival, growth, and differentiation of neuronal populations (Numakawa et al., 2010). Therefore, in a therapeutic perspective, effective overexpression of such essential neurotrophic factors is the key to curing the disease without causing severe and prolonged pathogenesis. Increased passage of MSCs naturally boost secretion of BDNF; MSCs are promising delivery systems for therapeutics in various neurodegenerative disorders, such as Alzheimer's disease and Parkinson's disease, because of their secretomes (Rodrigues Hell et al., 2009; Harrell et al., 2021; Jalali et al., 2021; Kuo et al., 2021). Therefore, BDNF-MSCs could be effective in treating RTT, because of their ability to overexpress BDNF and the presence of neuroprotective or immunomodulatory exosomes.

To investigate the therapeutic effects of BDNF/BDNF-MSCs on RTT, we performed parallel experiments on MECP2-silenced neuroblastoma cells (SHSY-5Y) and MECP2 deficient mice. In both experiments, we injected BDNF-MSCs in conjunction with a PTP1B inhibitor. PTP1B is a major metabolic regulator that attenuates insulin and leptin signaling. PTP1B levels are increased in mouse models of RTT (Krishnan et al., 2015; Fukuhara et al., 2019; Köhn, 2020). PTP1B targets tropomyosinrelated kinase $\mathrm{B}$ (TRKB; the receptor for BDNF) and negatively regulates BDNF signaling (Ozek et al., 2014). Therefore, we used a PTP1B inhibitor to prevent the loss of BDNF signaling. To further investigate the mechanisms underlying the enhancement of neuronal populations, the effects of BDNF on the activation of AKT and ERK1/2 survival signaling and p38 apoptosis signaling, were evaluated, with respect to the expression levels of BDNF and MECP2. Both in vivo and in vitro studies using the PTP1B inhibitor showed that BDNF/BDNF-MSCs induced the activation of TRKB; the upstream signaling pathways, including ERK1/2 and AKT, were activated. However, the p38 pathway, which is a signaling pathway downstream of the interaction between TRKB and BDNF, was inhibited. There were significant differences among the expression levels of these upstream and downstream cascades, between salineinjected and BDNF/BDNF-MSC-injected groups, indicating that BDNF activates the TRKB receptor after blocking PTP1B. Among the studied cascades, ERK1/2 is known for its role in the expression of synaptic proteins and RTT is considered a synaptic development disorder caused by the dysfunction 
of MECP2 (Johnston et al., 2003; Kumamaru et al., 2011). Recent animal study shown that TRKB plays crucial role in restoration of hippocampal synaptic plasticity in MECP2 mutant mice ( $\mathrm{Li}$ et al., 2017). The findings in the present study also supported this finding, and it was observed that local injection of rhBDNF/BDNF-MSCs gradually increased neuronal numbers and cell synapses. Considerable improvements in synaptic plasticity and signaling were observed in CA2 of the hippocampus and striatum, while minor changes were observed in the cortex in the $M E C P 2$ knockout mice, in response to BDNF treatments. The difference in response could be explained by the distance from the initial injection site in the ventricle and the distribution rate of the injected therapeutic molecules. The striatum is the closest to the ventricle and the highest increases in the neural synapse was observed in this area. The strength of the synapse decreased in the hippocampus and cortex, which were farther from the injection site. In addition, cresyl violet staining indicated similar improvements in neurogenesis. The BDNF-injected groups exhibited increased neurogenesis, compared to the saline-injected group. The increase in cell numbers in the striatum was similar between the rhBDNF and BDNF-MSCs injected groups. However, the cell number significantly increased in the cortex and hippocampus of the BDNF-MSC-injected group. This could be attributed to the short half-life of BDNF. The increase in neuronal population was in the following series according to the distance from the initial injection point (ventricle): striatum $>$ hippocampus $>$ cortex in regard with treatment of BDNF/BDNF-MSC. The body weights of the mice were measured at the first and second week following BDNF/BDNF-MSC treatment and there were losses observed in the groups treated with saline and rhBDNF injections. However, the administration of BDNF-MSCs resulted in weight gain. In addition, the highest size and weight of the brain was found in the BDNF-MSC-injected group, followed by the groups administered with rhBDNF and saline.

\section{CONCLUSION}

In conclusion, the local injection of rhBDNF or BDNF-MSCs in conjunction with the inhibition of PTP1B in MECP2 deficient

\section{REFERENCES}

Abuhatzira, L., Makedonski, K., Kaufman, Y., Razin, A., and Shemer, R. (2007). MeCP2 deficiency in the brain decreases BDNF levels by REST/CoRESTmediated repression and increases TRKB production. Epigenetics 2, 214-222. doi: 10.4161/epi.2.4.5212

Bayarsaikhan, D., Bayarsaikhan, G., and Lee, B. (2020). AGE-RAGE System and its Application in Stem Cell Therapy. J. Stem Cell Res. Dev. Ther. 7:060. doi: 10.24966/SRDT-2060/100060

Collins, B. E., Merritt, J. K., Erickson, K. R., and Neul, J. L. (2021). Safety and efficacy of genetic MECP2 supplementation in the R294X mouse model of Rett syndrome. Genes Brain Behav. doi: 10.1111/gbb.12739 [Epub Online ahead of Print].

Damen, D., and Heumann, R. (2013). MeCP2 phosphorylation in the brain: from transcription to behavior. Biol. Chem. 394, 1595-1605. doi: 10.1515/hsz-20130193 mice could lead to increase neurogenesis and syanpses through BDNF-dependent mechanisms such as ppAKT and ppERK 1/2. This led to increased brain weight, reduced loss in body weight and improved survival in RTT mice. Althogh, further studies are required for ascertaining the safe and effective dosage of BDNFMSC and methods for delivering BDNF-MSC, BDNF-MSC treatment is a promising therapeutic strategy for treating RTT.

\section{DATA AVAILABILITY STATEMENT}

The raw data supporting the conclusions of this article will be made available by the authors, without undue reservation.

\section{ETHICS STATEMENT}

The animal study was reviewed and approved by Animal Center Ethics Board, Center of Animal Care and Use, Lee Gil Ya Cancer and Diabetes Institute of GACHON University, (LCDI-2018-008).

\section{AUTHOR CONTRIBUTIONS}

BL contributed to the conception and design of the study. HK and DB performed data analysis and wrote the manuscript. DB, $\mathrm{HK}$, JL, and GB performed the experiments and participated in drafting the manuscript. All authors read and approved the final manuscript.

\section{FUNDING}

This research was supported by the Bio \& Medical Technology Development Program of the National Research Foundation (NRF) funded by the Korean government (MSIT) (No. 2017M3A9B4061408).

Fukuhara, S., Nakajima, H., Sugimoto, S., Kodo, K., Shigehara, K., Morimoto, H., et al. (2019). High-fat diet accelerates extreme obesity with hyperphagia in female heterozygous Mecp2-null mice. PLoS One 14:e0210184. doi: 10.1371/ journal.pone.0210184

Gao, M., Lu, P., Lynam, D., Bednark, B., Campana, W. M., Sakamoto, J., et al. (2016). BDNF gene delivery within and beyond templated agarose multichannel guidance scaffolds enhances peripheral nerve regeneration. J. Neural. Eng. 13:066011. doi: 10.1088/1741-2560/13/6/066011

Harrell, C. R., Volarevic, A., Djonov, V., and Volarevic, V. (2021). Mesenchymal Stem Cell-Derived Exosomes as New Remedy for the Treatment of Neurocognitive Disorders. Int. J. Mol. Sci. 22:1433. doi: 10.3390/ijms22031433

Jalali, M. S., Saki, G., Farbood, Y., Azandeh, S. S., Mansouri, E., Ghasemi Dehcheshmeh, M., et al. (2021). Therapeutic effects of Wharton's jelly-derived Mesenchymal Stromal Cells on behaviors, EEG changes and NGF-1 in rat model of the Parkinson's disease. J. Chem. Neuroanat. 113:101921. doi: 10.1016/ j.jchemneu.2021.101921 
Johnston, M. V., Mullaney, B., and Blue, M. E. (2003). Neurobiology of Rett syndrome. J. Child. Neurol. 18, 688-692. doi: 10.1177/08830738030180100501

Khalin, I., Alyautdin, R., Wong, T. W., Gnanou, J., Kocherga, G., and Kreuter, J. (2016). Brain-derived neurotrophic factor delivered to the brain using poly (lactide-co-glycolide) nanoparticles improves neurological and cognitive outcome in mice with traumatic brain injury. Drug Deliv. 23, 3520-3528. doi: $10.1080 / 10717544.2016 .1199609$

Köhn, M. (2020). Turn and Face the Strange: a New View on Phosphatases. ACS Cent Sci. 6, 467-477. doi: 10.1021/acscentsci.9b00909

Krishnan, N., Krishnan, K., Connors, C. R., Choy, M. S., Page, R., Peti, W., et al. (2015). PTP1B inhibition suggests a therapeutic strategy for Rett syndrome. J. Clin. Invest. 125, 3163-3177. doi: 10.1172/JCI80323

Kumamaru, E., Numakawa, T., Adachi, N., and Kunugi, H. (2011). Glucocorticoid suppresses BDNF-stimulated MAPK/ERK pathway via inhibiting interaction of Shp2 with TrkB. FEBS Lett. 585, 3224-3228. doi: 10.1016/j.febslet.2011.09.010

Kuo, S. C., Chio, C. C., Yeh, C. H., Ma, J. T., Liu, W. P., Lin, M. T., et al. (2021). Mesenchymal stem cell-conditioned medium attenuates the retinal pathology in amyloid- $\beta$-induced rat model of Alzheimer's disease: underlying mechanisms. Aging Cell 20:e13340. doi: 10.1111/acel.13340

Lee, J., Bayarsaikhan, D., Bayarsaikhan, G., Kim, J. S., Schwarzbach, E., and Lee, B. (2020). Recent advances in genome editing of stem cells for drug discovery and therapeutic application. Pharmacol. Ther. 209:107501. doi: 10. 1016/j.pharmthera.2020.107501

Li, W., Bellot-Saez, A., Phillips, M. L., Yang, T., Longo, F. M., and Pozzo-Miller, L. (2017). A small-molecule TrkB ligand restores hippocampal synaptic plasticity and object location memory in Rett syndrome mice. Dis. Model Mech. 10, 837-845. doi: 10.1242/dmm.029959

Lombardi, L. M., Zaghlula, M., Sztainberg, Y., Baker, S. A., Klisch, J., Tang, A. A., et al. (2017). An RNA interference screen identifies druggable regulators of MeCP2 stability. Sci. Transl. Med. 9:eaaf7588. doi: 10.1126/scitranslmed. aaf7588

Lopes, C. D. F., Gonçalves, N. P., Gomes, C. P., Saraiva, M. J., and Pêgo, A. P. (2017). BDNF gene delivery mediated by neuron-targeted nanoparticles is neuroprotective in peripheral nerve injury. Biomaterials 121, 83-96. doi: 10. 1016/j.biomaterials.2016.12.025

Luoni, M., Giannelli, S., Indrigo, M. T., Niro, A., Massimino, L., Iannielli, A., et al. (2020). Whole brain delivery of an instability-prone Mecp2 transgene improves behavioral and molecular pathological defects in mouse models of Rett syndrome. Elife 9:e52629. doi: 10.7554/eLife.52629

Napoletani, G., Vigli, D., Cosentino, L., Grieco, M., Talamo, M. C., Lacivita, E., et al. (2021). Stimulation of the Serotonin Receptor 7 Restores Brain Histone H3 Acetylation and MeCP2 Corepressor Protein Levels in a Female Mouse Model of Rett Syndrome. J. Neuropathol. Exp. Neurol. 80, 265-273. doi: 10.1093/jnen/ nlaa158

Numakawa, T., Suzuki, S., Kumamaru, E., Adachi, N., Richards, M., and Kunugi, H. (2010). BDNF function and intracellular signaling in neurons. Histol. Histopathol. 25, 237-258. doi: 10.14670/HH-25.237
Ozek, C., Kanoski, S. E., Zhang, Z. Y., Grill, H. J., and Bence, K. K. (2014). Proteintyrosine phosphatase 1B (PTP1B) is a novel regulator of central brain-derived neurotrophic factor and tropomyosin receptor kinase B (TrkB) signaling. J. Biol. Chem. 289, 31682-31692. doi: 10.1074/jbc.M114.603621

Pejhan, S., and Rastegar, M. (2021). Role of DNA Methyl-CpG-Binding Protein MeCP2 in Rett Syndrome Pathobiology and Mechanism of Disease. Biomolecules 11:75. doi: 10.3390/biom11010075

Percy, A. K., Neul, J. L., Glaze, D. G., Motil, K. J., Skinner, S. A., Khwaja, O., et al. (2010). Rett syndrome diagnostic criteria: lessons from the Natural History Study. Ann. Neurol. 68, 951-955. doi: 10.1002/ana.22154

Riikonen, R. (2003). Neurotrophic factors in the pathogenesis of Rett syndrome. J. Child Neurol. 18, 693-697. doi: 10.1177/08830738030180101101

Rodrigues Hell, R. C., Silva Costa, M. M., Goes, A. M., and Oliveira, A. L. (2009). Local injection of BDNF producing mesenchymal stem cells increases neuronal survival and synaptic stability following ventral root avulsion. Neurobiol. Dis. 33, 290-300. doi: 10.1016/j.nbd.2008.10.017

Tsai, S. J. (2012). Peripheral administration of brain-derived neurotrophic factor to Rett syndrome animal model: a possible approach for the treatment of Rett syndrome. Med. Sci. Monit. 18, HY33-HY36. doi: 10.12659/msm.883 251

Vanhala, R., Korhonen, L., Mikelsaar, M., Lindholm, D., and Riikonen, R. (1998). Neurotrophic factors in cerebrospinal fluid and serum of patients with Rett syndrome. J. Child Neurol. 13, 429-433. doi: 10.1177/088307389801300903

Vigli, D., Cosentino, L., Pellas, M., and De Filippis, B. (2021). Chronic Treatment with Cannabidiolic Acid (CBDA) Reduces Thermal Pain Sensitivity in Male Mice and Rescues the Hyperalgesia in a Mouse Model of Rett Syndrome. Neuroscience 453, 113-123. doi: 10.1016/j.neuroscience.2020.09.041

Villard, L. (2007). MECP2 mutations in males. J. Med. Genet. 44, 417-423. doi: 10.1136/jmg.2007.049452

Conflict of Interest: The authors declare that the research was conducted in the absence of any commercial or financial relationships that could be construed as a potential conflict of interest.

Publisher's Note: All claims expressed in this article are solely those of the authors and do not necessarily represent those of their affiliated organizations, or those of the publisher, the editors and the reviewers. Any product that may be evaluated in this article, or claim that may be made by its manufacturer, is not guaranteed or endorsed by the publisher.

Copyright (๑) 2021 Kim, Bayarsaikhan, Lee, Bayarsaikhan and Lee. This is an openaccess article distributed under the terms of the Creative Commons Attribution License (CC BY). The use, distribution or reproduction in other forums is permitted, provided the original author(s) and the copyright owner(s) are credited and that the original publication in this journal is cited, in accordance with accepted academic practice. No use, distribution or reproduction is permitted which does not comply with these terms. 